\title{
Compreensão de profissionais de enfermagem sobre os benefícios e complicações da hipodermóclise em pacientes acometidos pelo câncer
}

\author{
Understanding nursing professionals about the benefits and complications of \\ hypodermoclysis in patients affected by cancer
}

\begin{abstract}
Entender a los profesionales de enfermería sobre los beneficios y las complicaciones de la hipodermocílisis en pacientes afectados por el cáncer
\end{abstract}

Anne Caroline Pereira Bezerra ${ }^{1 *}$, Gilvânia Smith da Nóbrega Morais ${ }^{1}$, Xênia Sheila Barbosa Aguiar Queiroz ${ }^{1}$, Alan Dionizio Carneiro ${ }^{1}$, José Rocha Gouveia Neto ${ }^{1}$, Larissa Hosana Paiva de Castro ${ }^{1}$.

\section{RESUMO}

Objetivo: Investigar a compreensão dos profissionais de enfermagem sobre os benefícios e complicações da hipodermóclise na terapêutica de pacientes acometidos pelo câncer avançado. Métodos: Trata-se de uma pesquisa de campo de abordagem qualitativa com análise temática. Participaram da investigação 11 profissionais de enfermagem que atuam em um serviço de referência em oncologia localizada na cidade de Campina Grande - PB. O período de coleta de dados transcorreu durante o mês de junho de 2018. Para coleta dos dados utilizou-se uma pesquisa semiestruturada. O estudo foi aprovado por Comitê de Ética em Pesquisa. Resultados: Os profissionais relataram que o favorecimento da qualidade de vida, eficácia no controle da dor, segurança na absorção de drogas, diminuição do risco de complicações e facilidade da técnica são os benefícios da via subcutânea. Além de apontar a falha na implementação da técnica como um determinante para as complicações relacionadas à via subcutânea. Conclusão: Devido ao conhecimento escasso sobre a Hipodermóclise, torna-se necessário investir na educação permanente em saúde, voltada para a capacitação desses profissionais sobre o tema, além da criação de protocolos que abordem o assunto, visando uma melhora na qualidade da assistência prestada e assegurando maior eficácia desses cuidados em saúde.

Palavras-chave: Cuidados paliativos, Enfermagem, Hipodermóclise.

\begin{abstract}
Objective: To investigate the understanding of nursing professionals about the benefits and complications of hypodermoclysis in the treatment of patients affected by advanced cancer. Methods: This is a field research with a qualitative approach with thematic analysis. Eleven nursing professionals who work in a referral service in oncology located in the city of Campina Grande - PB participated in the investigation. The data collection period took place during the month of June 2018. A semi-structured survey was used to collect the data. The study was approved by the Research Ethics Committee. Results: Professionals reported that favoring quality of life, effective pain control, safe drug absorption, reduced risk of complications and ease of technique are the benefits of the subcutaneous route. In addition to pointing out the failure to implement the technique as a determinant for complications related to the subcutaneous route. Conclusion: Due to the scarce knowledge about Hypodermoclysis, it is necessary to invest in permanent health education, focused on the training of these professionals on the subject, in addition to the creation of protocols that address the subject, aiming at improving the quality of care provided and ensuring greater effectiveness of this health care.
\end{abstract}

Keywords: Palliative care, Nursing, Hypodermoclysis.

\footnotetext{
${ }^{1}$ Universidade Federal de Campina Grande (UFCG), Campina Grande - PB.

*E-mail: anne.carolinepb@outlook.com
} 


\section{RESUMEN}

Objetivo: Investigar la comprensión de los profesionales de enfermería sobre los beneficios y complicaciones de la hipodermoclisis en el tratamiento de pacientes afectados por cáncer avanzado. Métodos: Esta es una investigación de campo con un enfoque cualitativo con análisis temático. Once profesionales de enfermería que trabajan en un servicio de referencia en oncología ubicado en la ciudad de Campina Grande - PB participaron en la investigación. El período de recopilación de datos tuvo lugar durante el mes de junio de 2018. Se utilizó una encuesta semiestructurada para recopilar los datos. El estudio fue aprobado por el Comité de Ética de Investigación. Resultados: Los profesionales informaron que favorecer la calidad de vida, el control efectivo del dolor, la absorción segura de medicamentos, la disminución del riesgo de complicaciones y la facilidad de la técnica son los beneficios de la vía subcutánea. Además de señalar la falta de implementación de la técnica como determinante de complicaciones relacionadas con la vía subcutánea. Conclusión: Debido al escaso conocimiento sobre la hipodermoclisis, es necesario invertir en educación permanente en salud, con el objetivo de capacitar a estos profesionales en el tema, además de la creación de protocolos que aborden el tema, con el objetivo de mejorar la calidad de la atención brindada y asegurando una mayor efectividad de este cuidado de la salud.

Palabras clave: Cuidados paliativos, Enfermería, Hipodermoclisis.

\section{INTRODUÇÃO}

O cuidado paliativo é a forma de cuidar de pessoas impossibilitadas de uma cura terapêutica, ou seja, é realizado um cuidado holístico, buscando conforto, controle da dor e demais sintomas, prevenção de agravos e demais incapacidades, manutenção das pessoas e atividades significativas para o paciente, dentre demais cuidados na assistência de pessoas com cuidados paliativos. Pacientes acometidos pelo câncer avançado pertencem ao grupo daqueles pacientes que necessitam do cuidado paliativo, pois correntemente esses pacientes não tem cura da doença (GOMES ALZ e OTHERO MB, 2016).

Tendo em vista a singularidade dos pacientes com câncer avançado, torna-se necessário que os profissionais de saúde, através da interdisciplinaridade e transdisciplinaridade, demandem uma atenção maior e mais especifica para essas pessoas.

Assim, cabe a essas equipes de saúde a busca de conhecimentos sobre técnicas em saúde, que garantam melhorias em sua prática profissional e visem à promoção do bem-estar desses doentes, aumentando sua qualidade de vida, e concomitantemente, diminuindo o sofrimento desencadeado pela doença (FERREIRA EAL, et al, 2019).

Nessa perspectiva, uma das opções existentes para a assistência nos cuidados paliativos, é a hipodermóclise: técnica que repõe fluídos e administra medicamentos por via subcutânea quando não há viabilidade de administração por outro meio, seja devido a fragilidade vascular ou impossibilidade do acesso venoso. Destacando-se por ser um recurso seguro, com pequeno risco de infecção e por ser simples, apresentando melhor custo benefício quando comparada a outros recursos (GODINHO NC e SILVEIRA LVA, 2017).

A hipodermóclise, como sendo uma via de administração de medicamento e fluidos, segura, confiável, de fácil manipulação e que aumenta o bem estar do paciente, já que quando comparado a outras vias de administração, gera o mínimo de desconforto e dor. É importante ressaltar que apesar de tantos benefícios, essa técnica ainda é subutilizada pelos profissionais de saúde, seja pela falta de capacitação desses ou pela indisponibilidade de protocolos que os orientem, e incentivem a utilização dessa terapêutica (JUSTINO ET, et al., 2013; NOVELLI BT, et al., 2019).

Considerando, conforme Parecer do COREN - SP n 031/2014 e COREN - BA n 004/2017, que a utilização desse procedimento, seja durante a punção e/ou administração de fluidos e medicamentos, podem ser realizadas tanto pelo enfermeiro quanto pelo técnico ou auxiliar de enfermagem, desde que estejam devidamente seguros sobre a técnica e seus cuidados. 
Além disso, considerando a sua utilidade no controle dos sintomas no final de vida, surgiu o interesse de realizar uma pesquisa mediada pelo seguinte questionamento: Qual a compreensão de profissionais de enfermagem sobre os benefícios e complicações da hipodermóclise em pacientes acometidos pelo câncer avançado?

Posto isto, o presente estudo tem como objetivos investigar a compreensão dos profissionais de enfermagem sobre os benefícios da hipodermóclise em pacientes sob cuidados paliativos e pesquisar a compreensão dos profissionais de enfermagem acerca das complicações da hipodermóclise em pacientes acometidos pelo câncer avançado.

\section{MÉTODOS}

Trata-se de uma pesquisa de campo de abordagem qualitativa transversal, do tipo exploratório e descritivo realizada em um hospital escola, localizado no agreste paraibano na cidade de Campina Grande. O período de coleta de dados transcorreu durante o mês de junho de 2018. Foram entrevistados 11 profissionais de Enfermagem que atuam na assistência direta aos pacientes oncológicos em cuidados paliativos.

Foi considerado como critério de inclusão ser funcionário efetivo desse serviço de saúde; desempenhar cuidado direto a pacientes oncológicos e/ou em estágio avançado; desenvolver suas atividades laborativas no período diurno; e atuar no serviço há pelo menos 1 ano. Foram excluídos os trabalhadores que não estavam presentes no período da coleta dos dados, devido a atestado, licença ou férias e aqueles que não atendiam aos critérios de inclusão.

Para coleta dos dados utilizou-se uma pesquisa semiestruturada, a qual foi norteada por um questionário sociodemográfico elaborado previamente pelos autores, sendo subdivido em três momentos, sendo esses: 1) perfil pessoal (identificação) e 2) profissional (tempo de formação e atuação na área); 3) questões subjetivas pertinentes ao objetivo de investigação.

Como ferramenta metodológica para análise dos dados, foi utilizado a técnica de análise de conteúdo de Bardin, composta por quatro etapas: Organização da análise ordenado em três polos: 1) pré-análise que correspondeu a fase da organização propriamente dita, em que se escolheu os documentos a serem submetidos a análise e a formulação das hipóteses para a interpretação final; 2) exploração do material: administração sistemática das decisões tomadas; 3) tratamento dos resultados, inferência e a interpretação: síntese e seleção dos resultados; Codificação que consistiu no processo de transformação dos dados brutos de forma sistemática; Categorização que perpassou pela classificação e reagrupamento das unidades de registro em número reduzido de categorias, deixando compreensível a massa de dados e sua diversidade; e por último, a Inferência, centrada nos aspectos implícitos da mensagem analisada (BARDIN L, 2011).

Vale ressaltar que o estudo obteve previa aprovação do Comitê de Ética em Pesquisa com Seres humanos da Universidade Federal de Campina Grande (UFCG), assim como, atendeu aos princípios éticos e as exigências presentes na resolução nํ466, de 12 de dezembro de 2012 do Ministério da Saúde.

Com isso, a participação dos profissionais se iniciou após assinatura do Termo de Consentimento Livre e Esclarecido. É importante destacar que o presente estudo foi submetido ao Comitê de Ética e Pesquisa da Universidade Federal de Campina Grande sendo aprovado sob CAAE no $=87757218.6 .0000 .5182$.

\section{RESULTADOS}

A amostra constituiu-se de 11 profissionais de enfermagem, sendo todos do sexo feminino, a maioria eram técnicos de enfermagem (54,5\%), e os demais enfermeiros (45,5\%). Em relação à atuação profissional, apenas $27,2 \%$ dos entrevistados relataram algum tipo de especialização voltada para oncologia e/ou pediatria, 90,9\% apontaram que durante a formação superior ou técnica tiveram acesso a informações sobre a administração de medicamentos por via subcutânea, e todos os profissionais relataram que após tal formação acadêmica não tiveram esse tipo de capacitação voltada para aos cuidados de enfermagem com pacientes oncológicos. 
Em relação ao tempo de atuação com pacientes oncológicos: 50\% (2 - 5 anos), 40\% (6 - 10 anos) e 10\% (>10 anos), já no tocante a atuação na enfermagem observou-se que: 45\% (21 - 30 anos), 27\% (4- 10 anos), 9\% (11- 20 anos) 19\% (> 30 anos). Ademais, a faixa etária dos participantes variou entre 28 e 55 anos, sendo: $45 \%$ (46 - 55 anos), $30 \%$ (28 - 35 anos), 20\% (36 - 45 anos) e 5\% (> 55 anos).

A partir dos discursos desses profissionais, emergiram-se duas categorias para análise, a saber: 1) Favorecimento da qualidade de vida, eficácia no controle da dor, segurança na absorção de drogas, diminuição do risco de complicações e facilidade da técnica como benefícios da via subcutânea; 2) Falha na implementação da técnica como determinante para complicações relacionadas à via subcutânea;

Favorecimento da qualidade de vida, eficácia no controle da dor, segurança na absorção de drogas, diminuição do risco de complicações e facilidade da técnica como benefícios da via subcutânea

Durante a fase de escolha para traçar os caminhos de uma terapêutica, a equipe deve levar em consideração os benefícios e complicações de cada procedimento que será utilizado, partindo desse princípio, alguns profissionais compreendem os benefícios da via subcutânea na oncologia, incluindo o favorecimento da qualidade de vida e o controle da dor nesses pacientes. Esta ideia encontra-se ratificada nas falas que seguem abaixo:

"[...] ultimamente eles estão usando muito o portocath, justamente para dar mais qualidade de vida e evitar tantas furadas nos pacientes né, principalmente quando estão acometidos por uma gravidade maior, para a gente dar certo conforto, mas no caso do portocath não pode ser feito para a hemotransfusão" (entrevistada 1)

"Assim, de benefício eu acho que é uma questão assim [...] é a questão da dor é, assim..." (entrevistada 2)

Apesar de equívocos quanto à capacidade de absorção de uma medicação administrada pela via subcutânea, os discursos evidenciam indiretamente a segurança na absorção de drogas administradas por hipodermóclise.

Alguns entrevistados compreendem os benefícios da via subcutânea a partir de suas experiências profissionais, mas não voltadas para pacientes oncológicos. Expressam em suas falas que a via SC diminui o risco de complicações em comparação à via $E V$ bem como revelam que se trata de uma técnica fácil de ser realizada. Tais fatos podem ser identificados nas falas a seguir:

"Os benefícios... é a questão da absorção que é a mais lenta diminuindo assim o risco né... de alguma complicação, como no caso se fosse endovenosa poderia ter alguma reação mais rápida ne... e mais prejudicial pra eles [...]" (entrevistada 3)

"Os benefícios é porque assim, a absorção pode ser mais lenta, mas é mais complicado do que é a endovenosa né [...]" (entrevistada 4)

"O benefício é evitar as doenças vasculares né isso, e a absorção que é bem mais rápido com um efeito satisfatório bem mais rápido do que na via (pausa)... é além de ser mais rápido ele é mais eficaz, ele absorve melhor porque na venosa como a circulação é volátil e a gente tem um nível de volemia muito alto, então muitas drogas não é absorvida e é eliminada sem ser absorvida.... e a subcutânea não, ela absorve melhor." (entrevistada 5)

"Os benefícios são fácil acesso, né... é fácil, dependendo da pele do paciente [...]" (entrevistada 6)

Apesar dos benefícios evidenciados por alguns participantes da pesquisa, relatos asseveram 0 desconhecimento dos benefícios da via subcutânea, sejam em pacientes com câncer avançado ou com outras patologias. Como observado nos relatos abaixo: 


\begin{abstract}
"[...] os benefícios eu não sei!" (entrevistada 7)
"Não conheço nenhum benefício da via subcutânea em relação as outras." (entrevistada 8)

"Também não sei né, se, se tem um maior benefício né, se é melhor do que administração endovenosa... Né, então assim todos os analgésicos aqui são via endovenosa, mesmo, então não tem rotina de médico e consequentemente a equipe de enfermagem realizar administração via subcutânea em paciente oncológico certo?!" (entrevistada 9)
\end{abstract}

\title{
Falha na implementação da técnica como determinante para complicações relacionadas à via subcutânea.
}

Questionados quanto às complicações envolvendo a via subcutânea, os participantes do estudo apesar de mencionarem que estas estão relacionadas principalmente aos cuidados de enfermagem que envolvem o local da punção e a implementação correta da técnica, não direcionam o discurso para os pacientes oncológicos, como pode ser observado nos relatos abaixo.

"As complicações né, no caso é se for muito profundo a subcutânea pode dar Flebite e também se for muito perto, com é (pausa) se for muito curta pode ficar no tecido intradérmico ai pode também causa lesão. Não conheço nenhum paciente que teve essas complicações aqui não. (entrevistada8)"

"[...] e de complicações acredito que pode haver porque é um tecido que embora seja pouco vascularizado, mas pode ocorrer a formação de algum abscesso, acredito, acho que só... se não for feito na via, no ângulo correto e no tecido correto." (entrevistada 2)

"[...] as complicações é a questão do local que se for administrado várias vezes em um só local pode causar hematomas, necrose, edema, inflamação, hiperemia." (entrevistada 3)

"O que conheço sobre as complicações é que se assim.... se a gente não fizer o rodízio ele pode causar a necrose tecidual, por isso a necessidade de fazer o rodízio[...]" (entrevistada 7 )

"[...] as complicações é a mesma que disse agora, pode apresentar edema, abscessos [...] inclusive teve um paciente aqui, que ficou bem assim, com o abscesso... depois de ser realizado [....]" (entrevistada 4)

"Eu sei que tem complicação para paciente que usam Insulina né, aquela complicação que pode ocorrer de ficar roxo, eu esqueci até o nome agora... mais que se fizer várias vezes em um local só pode ficar com hematoma, só. Mas de outras medicações como o Bricanyl eu nunca vi complicação, só mais paciente em uso de Insulina." (entrevistada 10)

"[...] e em relações as complicações têm que fazer o rodízio das áreas onde se administra para evitar que tenham um abcesso, as complicações são essas né." (entrevistada 6)

Alguns relatos demonstram o desconhecimento dos profissionais inseridos nesta pesquisa quanto as complicações da via subcutânea, sejam em pacientes com câncer avançado ou com outras patologias e evidenciam novamente o desuso da via SC nesses serviços. Conforme evidenciam os relatos abaixo.

"[...] E as complicações que conheço é relacionada a cateter, porque aqui é raríssimo medicação por via subcutânea no nosso paciente. Geralmente aqui a gente só conhecer mais o caso do Filgrastim por essa via." (entrevistada 1)

"Não conheço nenhuma complicação por essa via." (entrevistada 2) 


\section{DISCUSSÃO}

Ao analisar o perfil dos profissionais participantes, nota-se que apesar de atuarem a mais de 2 anos em oncologia, apenas uma pequena parcela apresentou especialização nessa área profissional. Esse fator acaba por interferir diretamente no número, ainda menor, de profissionais que tiveram acesso a informações sobre a administração de medicamentos por via subcutânea (SC), além da totalidade que após sua formação acadêmica, não teve acesso a cursos de capacitações sobre esse tema.

Infelizmente, esses dados demonstram um déficit, ainda existente entre os profissionais, em relação a não busca de novos conhecimentos sobre a sua área de atuação, indicando uma carência na educação permanente em saúde, a qual visa a qualificação desses trabalhadores como forma de melhorar suas práticas profissionais e o atendimento ao público (ALMEIDA JRS, et al., 2016).

Sendo assim, a promoção da qualidade de vida dos pacientes com câncer avançado deve ser um dos objetivos das capacitações voltadas para os cuidados de enfermagem, sendo prestados de forma qualificada, digna, holística e individualizada.

Posto isso, esse profissional deve estar atento às informações sobre procedimentos como a hipodermóclise, que assim como refere a entrevistada 2, está relacionada ao alivio da dor desses pacientes, e a qual entrevistada 1 entende ser uma técnica que oferecer mais conforto ao doente.

Logo, a hipodermóclise oferece o mínimo de desconforto por eliminar a necessidade de injeções frequentes, atuando assim na diminuição da dor causada pelas repetidas punções venosas, necessárias para infusão dos medicamentos e fluidos (MARTINS AKC, et al., 2017; ROCHA AFP, et al., 2015; AZEVEDO DL, 2016).

Por conseguinte, a principal característica que um fármaco deve possuir para ser administrado por via subcutânea é ser solúvel em água, isso se deve ao baixo poder irritante e menor risco de acúmulo desses medicamentos no tecido, dessa forma, todas as substâncias administradas nessa via devem estar na forma líquida ou diluídos, sendo a diluição de pelo menos 100\% (CHIRIVELLA CM, et al., 2015; GODINHO NC e SILVEIRA LVA, 2017). Além disto, a dose infundida na via SC deve ser menor do que a administração por via oral (AZEVEDO DL, 2016).

A capacidade de absorção do tecido SC possibilita o recebimento de fluídos e medicamentos por esta via, principalmente pelos seus componentes estruturais, como densas conexões e tecido adiposo, sistema linfático, glândulas e nervos, além de capilares sanguíneos. Dessa forma, se torna possível a absorção e circulação dos fármacos no espaço SC, a partir dos mecanismos de difusão e perfusão, ultrapassando apenas as células endoteliais (FERREIRA EAL, et al., 2019).

Vale ressaltar que na via SC a concentração sérica máxima é obtida em tempo maior do que para as demais vias parenterais mais comuns, como a Intravenosa (IV) e intramuscular (IM). Além disso, a via SC possui uma absorção lenta (ANCP, 2013) em oposição ao discurso da entrevistada 5, por esse motivo a SC é indicada principalmente para os casos em que se deseja uma ação continua da medicação no organismo.

Um estudo avaliou a via de administração mais utilizada em um serviço de atendimento ambulatorial de quimioterapia em um hospital público do Sul do Brasil, no período de junho a agosto de 2016, constatando que as três primeiras vias mais utilizadas, mediante as prescrições, foram a endovenosa (EV) $(48,5 \%)$, endovenosa e via oral (31,1\%), subcutânea (10,5\%) (CAROLLO JB, et al., 2017).

Este resultado ratifica os discursos dos participantes da presente pesquisa e de outros estudos, os quais demonstram a subutilização da via subcutânea nos serviços de saúde e o crescente uso da EV (JUSTINO ET, et al., 2013; NOVELLI BT, et al., 2019; GOMES NS, et al., 2017).

Como relatado pela entrevistada 1, o cateter totalmente implantado (portocath) vem sendo frequentemente utilizado na oncologia. Trata-se de um dispositivo agulhado que possibilita a administração de vastos grupos farmacológicos, especialmente drogas e quimioterápicos antineoplásicos, proporcionando segurança quanto a extravasamento e evitando a ação vesicante ou irritante dessas drogas no sistema circulatório. 
Outro fator positivo atribuído ao dispositivo é a capacidade de melhorar a qualidade de vida desses pacientes, por proporcionar maior bem-estar durante a administração dos fármacos, menores riscos das exposições relacionadas as manipulações e infecções, quando comparado as demais ferramentas de infusões (ZERATI AE, et al., 2017).

Se por um lado, alguns autores esclarecem que o portocath pode ser método de escolha para transfusão e coleta de sangue, infusão de dieta via parenteral, administração de medicamentos como antibióticos, analgésicos, e em especial, quimioterápicos (HONÓRIO RPP, et al., 2011).

Pesquisadores afirmam que o cateter totalmente implantado não é indicado para infusão de grandes volumes de fluidos, nem para hemotransfusões ou coleta de sangue (exceto hemoculturas), devido ao seu pequeno calibre, o que favorece a obstrução, aumentando assim as chances da perda do dispositivo, indo a favor do discurso da entrevistada 1, que afirma que o portocath não pode ser utilizado para a administração de hemotransfusão (THEOBALD MR, 2014; CCIH, 2013).

Salienta-se que diante de um acesso venoso difícil, além do cateter totalmente implantado, a hipodermóclise é considerada como sendo a via parenteral mais acessível e mais confortável que a venosa. Dentre outras vantagens, a administração de drogas a partir do tecido subcutâneo pode ser realizada em qualquer ambiente de cuidado, inclusive no domicílio (sendo mais uma indicação para os Cuidados Paliativos), raramente provoca complicações locais, tem baixo risco de efeitos adversos sistêmicos (hiponatremia, hipervolemia, congestão), reduz a flutuação das concentrações plasmáticas de medicamento, incluindo os opióides, de modo que auxilia no controle efetivo da dor, como revelado nos discursos dos participantes dessa pesquisa, além disso, possui baixo custo e está relacionada à maior facilidade para manejo da hidratação e maior segurança contra infecções locais, quando comparada a endovenosa (AZEVEDO DL, 2016; ZITELLI PMY, et al., 2014).

Nestes termos, alguns estudos realizados sobre a hipodermóclise, concluíram que essa técnica é segura, de baixo custo, pouco invasiva, de fácil inserção e manutenção, como verificado na fala da entrevistada 6 , possibilitando qualidade e conforto no tratamento sintomático (PONTALTI G, et al., 2016; VERAS GL, et al., 2014; NOVELLI BT, et al., 2019).

No que tange as complicações decorrentes da utilização da via subcutânea foi evidente no estudo um desconhecendo significativos entre os participantes. Este fato resulta em uma assistência deficiente para o paciente, tendo em vista que apesar de raras, a prevenção de complicações relacionadas a essa via dependem da habilidade técnica e do conhecimento do profissional de enfermagem (AZEVEDO DL, 2016; ASSIS BF, et al., 2018).

As complicações comuns relacionadas à hipodermóclise estão relacionadas ao local da punção e inclui desconforto, tromboflebite, septicemia, calor, eritema, edema, dor, inflamação, sangramento, formação de abcesso, celulite no sítio de infusão e necrose tecidual (BRAZ CL, et al., 2015; VERAS GL, et al., 2014; GODINHO NC e SILVEIRA LVA, 2017).

Ademais, um estudo realizado com objetivo de identificar, a partir de uma revisão integrativa, as literaturas sobre os efeitos adversos da hipodermóclise em adultos, concluiu que esses efeitos adversos não causam danos graves a esses pacientes, sendo de baixa incidência e similares a via endovenosa, e for fim, concorda que, quando implementada na assistência, essa terapia é efetiva e segura para a hidratação e analgesia, ressaltando que deve ser utilizada mediante análise da situação clínica do paciente (NUNES PMSA e SOUZA RCS, 2015).

Assim, conforme revelado pelos participantes, o profissional deverá estar atento aos locais recomendados para punção do tecido subcutâneo e realizar rodízio, ter atenção com o volume que irá infundir, além de efetuar uma antissepsia rigorosa da pele do paciente. Por isso, torna-se necessária a implantação de uma assistência sistematizada ao paciente que está em uso dessa terapia, prestando os cuidados de forma individualizada e com excelência (ASSIS BF, et al., 2018). 


\section{CONCLUSÃO}

Mediante resultados obtidos, considera-se que o objetivo da pesquisa foi alcançado, ao demonstrar os conhecimentos dos profissionais de enfermagem sobre os benefícios e complicações da hipodermóclise durante a terapêutica dos pacientes acometidos pelo câncer avançado, promovendo uma discussão sobre esses achados e enfatizando algumas lacunas no que se refere aos conhecimentos dos profissionais inseridos na investigação. Ademais a pesquisa contribuiu para difundir e aprimorar os conhecimentos desses trabalhadores sobre o assunto, enfatizando a necessidade quanto à criação de protocolos e de ações voltadas para a educação permanente, tendo em vista que a maior parte desses profissionais de enfermagem não possuíam conhecimentos concretos e ampliados sobre a utilização da hipodermóclise e da via subcutânea na sua área de atuação, o que pode dificultar as suas práticas profissionais e interferir negativamente no bem estar e no sofrimentos físico e mental dos seus pacientes. Por fim, ressalta-se a importância de novos estudos sobre o assunto, dada a sua importância para as boas práticas em saúde.

\section{REFERÊNCIAS}

1. ALMEIDA JRS, et al. Educação permanente em saúde: uma estratégia para refletir sobre o processo de trabalho. Revista da ABENO, 2016; 16(2): 07-15.

2. ACADEMIA NACIONAL DE CUIDADOS PALIATIVOS. Manual de Cuidados Paliativos ANCP. São Paulo. 2013.

3. ASSIS BF, et al. Assistência de enfermagem na hipodermóclise: um método antigo para o novo cuidado. In: Anais da 18 ${ }^{\text {a }}$ Semana de Pesquisa da Universidade Tiradentes, 2018; (18).

4. AZEVEDO DL. O uso da via subcutânea em geriatria e cuidados paliativos. São Paulo. SBGG, 2016; 56p.

5. BARDIN L. Análise de conteúdo. Lisboa. 2011;229p.

6. BRAZ CL, et al. Administração de medicamentos por hipodermóclise: uma revisão da literatura. Revista Brasileira de Farmácia Hospitalar e Serviços de Saúde, 2015; 6(1): 6-12.

7. CAROLLO JB, et al. Incidentes relacionados a medicamentos em um ambulatório de quimioterapia. Acta Paulista de Enfermagem. 2017; 30(4): 428-34.

8. CCIH. Manipulação de cateteres vasculares centrais de longa permanência. Rio de Janeiro, 2013.

9. CHIRIVELLA CM, et al. Administración de medicamentos por vía subcutánea en cuidados paliativos. Revista Farmacia Hospialaria, 2015; 39(2): 71-79.

10. CONSELHO REGIONAL DE ENFERMAGEM (Bahia). Parecer Coren - BA n004/2017. Competência técnica da equipe de enfermagem na realização da hipodermóclise.

11. CONSELHO REGIONAL DE ENFERMAGEM (São Paulo). Parecer Coren - SP $n^{0}$ 031/2014. Punção e administração de fluidos na hipodermóclise.

12. FERREIRA EAL, et al. Uso da via subcutânea em pediatria. São Paulo: ANCP, 2019.

13. GODINHO NC, SILVEIRA LVA. Manual de hipodermóclise. Hospital das Clínicas da Faculdade de Medicina de Botucatu - HCFMB. 2017; 34p.

14. GOMES ALZ, OTHERO MB. Cuidados paliativos. Estudos avançados, 2016; 30(88): 155-166.

15. GOMES NS, et al. Conhecimentos e práticas da enfermagem na administração de fluidos por via subcutânea. Revista Brasileira de Enfermagem. 2017; 70(5): 1155-1164.

16. HONÓRIO RPP, et al. Validação de procedimentos operacionais padrão no cuidado de enfermagem de pacientes com cateter totalmente implantado. Revista Brasileira de Enfermagem, 2011; 64(5): 882-889.

17. JUSTINO ET, et al. Hipodermóclise em pacientes oncológicos sob cuidados paliativos. Cogitare Enfermagem, 2013; $18(1) ; 84-89$.

18. MARTINS AKC, et al. A hipodermóclise como ferramenta para cuidados paliativos. Anais do Evento Even3, 2017.

19. NOVELLI BT, et al. Recomendações para utilização da hipodermóclise em pacientes sobre cuidados paliativos. Revista Enfermagem em Evidência, Bebedouro SP, 2019; 3(1): 139-153.

20. NUNES PMSA, SOUZA RCS. Efeitos adversos da hipodermóclise em pacientes adultos: revisão integrativa. Revista Mineira de Enfermagem, 2015; 20(4): 01-06.

21. PONTALTI G, et al. Benefícios da Hipodermóclise na Clínica Paliativa de Pacientes com Câncer: Relato de Caso. Revista Brasileira de Cancerologia, 2016; 62(3): 247-252.

22. ROCHA AFP, et al. O alívio da dor oncológica: estratégias contadas por adolescentes com câncer. Revista Texto Contexto Enfermagem, Florianópolis, 2015; 24(1): 96-104.

23. THEOBALD MR. Procedimento Operacional Padrão no Manejo de Cateter Totalmente Implantado. Mato Grosso do Sul. Universidade Federal de Santa Catarina, 2014; 1 -2.

24. VERAS GL, et al. Evidências clínicas no uso da hipodermóclise em pacientes oncológicos: revisão integrativa da literatura. Revista Eletrônica Gestão e Saúde, 2014; 5(1): 2877-2893.

25. ZERATI AE, et al. Cateteres venosos totalmente implantáveis: histórico, técnica de implante e complicações. J Vasc Bras, 2017;16(2): 128-139.

26. ZITELLI PMY, et al. Hipodermóclise no paciente oncológico em Cuidados Paliativos. Revista Saúde - UNG, 2014; 8(2): 37-43. 